Address for Correspondence: Monir Sadat Hakemi, Associate Professor, Nephrology Department Dr. Shariati Hospital, Tehran University of Medical Sciences, Tehran 1411713135, Iran

Email: mhakemi@tums.ac.ir

\begin{tabular}{|l|}
\hline Access this article online \\
\hline $\begin{array}{l}\text { Website: } \\
\text { www.intern-med.com }\end{array}$ \\
\hline $\begin{array}{l}\text { DOI: } \\
\text { 10.1515/tim-2016-0030 }\end{array}$ \\
\hline Quick Response Code: \\
\hline \\
\\
\\
\\
\hline
\end{tabular}

\title{
Association of serum intact fibroblast growth factor 23 with left ventricular mass and different echocardiographic findings in patients on hemodialysis
}

\author{
Amir Ahmad Nassiri ${ }^{1}$, Monir Sadat Hakemi ${ }^{2}$, Reza Safar-Pour ${ }^{1}$, Ali Ahmadi ${ }^{3}$, \\ Maryam Tohidi ${ }^{4}$, Babak Sharif Kashani ${ }^{5}$, Fatemeh Esfehani ${ }^{6}$, Soudabeh Alatab ${ }^{2}$ \\ 'Department of Nephrology, Masih Daneshvari Hospital, Shahid Beheshti University of Medical Sciences, \\ Tehran, Iran; \\ ${ }^{2}$ Department of Nephrology, Dr. Shariati Hospital, Tehran University of Medical Sciences, Tehran, Iran; \\ ${ }^{3}$ Department of Epidemiology, Shahid Beheshti University of Medical Sciences, Tehran, Iran; \\ ${ }^{4}$ Department of Pathology, Shahid Beheshti University of Medical Sciences, Tehran, Iran; \\ ${ }^{5}$ Department of Cardiology, Massih-Daneshvari Hospital, Shahid Beheshti University of Medical Sciences, \\ Tehran, Iran. \\ ${ }^{6}$ Research Center, Dr. Shariati Hospital, Tehran University of Medical Sciences, Tehran, Iran.
}

\section{ABSTRACT}

Objectives: To determine the association of fibroblast growth factor 23 (FGF23) with left ventricular hypertrophy (LVH) through the assessment of left ventricular (LV) mass and left ventricular mass index (LVMI) in patients on hemodialysis, this study was done. Methods: All patients on hemodialysis who are older than 18 years and in whom hemodialysis vintage was at least 6 months were enrolled. All patients were on hemodialysis thrice a week for $4 \mathrm{~h}$ using low-flux dialysis filters, polysulfone membranes, reverse osmosis purified water, and bicarbonate-base hemodialysis solution. The exclusion criteria were any respiratory illness or pulmonary infection, cigarette smoking, and the presence of pericarditis or pericardial effusion. Additionally, patients with a known coronary artery disease, any form of cardiac arrhythmias, any cardiomyopathy or severe valvular heart disease diagnosed by echocardiography, acute congestive heart failure (CHF), and acute myocardial infarction were not included. Echocardiography was conducted by an experienced operator for all the enrolled patients using the ACUSON SC2000 ${ }^{\mathrm{TM}}$ ultrasound system transducer (Siemens), with a frequency bandwidth of: $1.5-3.5 \mathrm{MHz}$. Patients were considered to have LVH if the LVMI was greater than $134 \mathrm{~g} / \mathrm{m}^{2}$ for men and greater than $110 \mathrm{~g} / \mathrm{m}^{2}$ for women. Results: A total of 61 patients ( 19 female and 42 male) were enrolled to the study. Mean ( \pm SD) age of the patients was 59.6 \pm 13.1 years. The median duration of hemodialysis was 23 (range: $6-120$ ) months. The median predialysis level of FGF23 was $1,977 \mathrm{pg} / \mathrm{mL}$ (range: $155-8,870$ ). LVH was seen in $73.8 \%$ of the patients $(n=45)$ and of them $66.7 \%$ were male. There was a statistically significant direct correlation between FGF23 and left ventricle diameter in end systole (LVDs) $(r=0.29, P=$ 0.027). However, the association of FGF23 with LV mass, LVMI, and left ventricular ejection fraction (LVEF) was not significant. Conclusion: This study does not show the correlation between FGF23 and LV mass in stable hemodialysis patients.

Key words: hemodialysis; left ventricular hypertrophy; fibroblast growth factor 23; echocardiography; intact parathyroid hormone

\section{INTRODUCTION}

Chronic kidney disease (CKD) is an international public health problem that increases the risk of premature death because of cardiovascular disease (CVD). Both atherosclerotic disease and congestive heart failure (CHF) are contributing to the higher rate of CVD in these patients, although the risk of $\mathrm{CHF}$ is more prominent. [1] Several studies regarding the CVDs in patients with CKD have shown that left ventricular hypertrophy $(\mathrm{LVH})$ is an important cause of CHF and arrhythmia 
and encountered as a strong risk factor for cardiovascular morbidity and mortality. ${ }^{[1,2]}$

Patients with CKD have a high prevalence of traditional cardiac risk factors ${ }^{[1,2]}$; however, the severity and the extent of their cardiovascular events seem to be disproportionate to these risk factors' profile. ${ }^{[1-3]}$ Studies have shown that metabolic bone disease is among the other factors contributing to the increased cardiovascular risk and reduced survival in patients with $\mathrm{CKD} \cdot{ }^{[3,4]}$ As all components of mineral metabolism (i.e., inorganic phosphate, calcium, parathyroid hormone, and vitamin D) affect each other, clarifying the true culprits responsible for cardiovascular events still remains a complex challenge.

Fibroblast growth factor 23 (FGF23) is the most recent discovered member of the fibroblast growth factors family. ${ }^{[4,5]}$ This circulating peptide hormone is released primarily by osteocytes and acts as an endocrine hormone regulating phosphate and vitamin $\mathrm{D}$ homeostasis through binding to fibroblast growth factor (FGF) receptor and single-pass transmembrane coreceptor of klotho located in the kidney and parathyroid glands. ${ }^{[4-6]}$ The main physiologic roles of this peptide include the reduction of reabsorption of filtered inorganic phosphate in the kidney proximal tubules and the promotion of phosphaturia by downregulation of the expression of sodium-phosphate cotransporters and the attenuation of systemic 1,25-dihydroxyvitamin D levels through decreased activation and enhanced degradation of 25-hydroxyvitamin $\mathrm{D}^{[5,6]}$ The effect of this peptide on parathyroid hormone (PTH) is still under debate. ${ }^{[0-8]}$

It has been shown that circulating levels of FGF23 increase gradually as CKD stage progresses and the renal capacity for phosphorus excretion declines. ${ }^{[9,10]}$ In end-stage renal disease, the serum levels of FGF23 are dramatically elevated compared to healthy individuals, raising up to 10,000-fold higher than normal population ${ }^{[10,11]}$.

Moreover, some articles have demonstrated that higher FGF23 levels are associated with greater risk of LVH. ${ }^{[10-12]}$ Faul and colleagues showed that FGF23 caused pathological hypertrophy of isolated rat cardiomyocytes via FGF receptor-dependent activation of the calcineurinNFAT signaling pathway, ${ }^{[13]}$ a finding that supports the causal role of elevated FGF23 in the pathogenesis of LVH. FGF23 is not only involved in the pathogenesis of CVD but also a marker of higher risk of $\mathrm{CHF} \cdot{ }^{[10,11,15]}$

It has been shown that FGF23 concentrations are elevated in patients who are on dialysis; ${ }^{[10-12]}$, however whether there is an association between FGF23 levels and LVH and other echocardiographic indexes of LV dysfunction in patients who are on dialysis is still unproven. ${ }^{[13,14,16]}$ Therefore, in this study, we decided to evaluate the role of FGF23 on LVH, left ventricular mass index (LVMI), and other echocardiographic findings in a group of patients on hemodialysis without known CVD.

\section{MATERIALS AND METHODS}

\section{Patients}

In this cross-sectional study, we enrolled 61 prevalent chronic hemodialysis patients, who underwent maintenance treatment thrice a week on hemodialysis section of Masih Daneshvari medical center affiliated to Shahid Beheshti University of Medical Sciences, Tehran, Iran, in 2014. The study protocol was approved by the ethic committee of Shahid Beheshti University of Medical Sciences. The research followed the tenets of the Declaration of Helsinki and Good Clinical Practice guidelines. All the necessary information was explained to patients, and informed consent was obtained before recruiting them into the study. All patients hemodialysis who were older than 18 years and in whom hemodialysis vintage was at least 6 months were enrolled. All patients were on hemodialysis thrice a week for $4 \mathrm{~h}$ using low-flux dialysis filters, polysulfone membranes, reverse osmosis purified water and bicarbonate-base hemodialysis solution.

The exclusion criteria were any respiratory illness or pulmonary infection, cigarette smoking, and the presence of pericarditis or pericardial effusion. Additionally, patients with a known coronary artery disease, any form of cardiac arrhythmias, any cardiomyopathy or severe valvular heart disease diagnosed by echocardiography, acute CHF, and acute myocardial infarction were excluded as well.

\section{Anthropometric and demographic data collection} Information on demographic characteristics, such as age, sex, medications, etiology of kidney disease, duration of hemodialysis, smoking status, coronary heart disease, myocardial infarction, as well as duration of diabetes and hypertension (HTN) were obtained from medical records.

After physical examination, weight, height and blood pressure were recorded. Systolic and diastolic blood pressures of the participants were assessed after resting for $10 \mathrm{~min}$. Blood pressures were measured through a conventional mercury manometer.

Body mass index (BMI) was calculated as the weight $(\mathrm{kg})$ divided by the square of the height $\left(\mathrm{m}^{2}\right)$. Body surface area (BSA) is assessed using the Dunois equation: BSA $\left(\mathrm{m}^{2}\right)=$ $0.007184 \times$ (height) $0.725 \times$ (weight) 0.425 , where height is expressed in meters and weight is in kilogram. ${ }^{[17]}$ 


\section{Echocardiographic assessment}

Echocardiography was conducted by an experienced operator for all the enrolled patients using the ACUSON SC2000 ${ }^{\mathrm{TM}}$ ultrasound system transducer (Siemens), with a frequency bandwidth of 1.5-3.5 MHz. M-mode and twodimensional measurements were performed in accordance with methods recommended by the American Society of Echocardiography. ${ }^{[18]}$ Measurements included fractional shortening (FS), left ventricle diameter in end diastole (LVDd), left ventricle diameter in end systole (LVDs), left ventricle volume in end diastole (LVEDV), left ventricle volume in end systole (LVESV), early to late ventricular filling velocity (E/A) ratio, and also posterior $L V$ wall thickness (PWT). Left ventricle ejection fraction (LVEF) was calculated from M-mode measurement. LV mass was calculated according to a formula described ${ }^{[18,19]}$ and indexed for BSA to give LVMI. Patients were considered to have LVH if the LVMI was greater than $134 \mathrm{~g} / \mathrm{m}^{2}$ for men and greater than $110 \mathrm{~g} / \mathrm{m}^{2}$ for women ${ }^{[19]}$.

\section{Laboratory assessments}

Blood samples were obtained after a long dialysisfree weekend interval before the next hemodialysis, after a minimum of 8-h overnight fast. Serum intact parathyroid hormone (iPTH) was measured by electrochemiluminescence immunoassay (ECLIA) using the kits and protocol from Roche Diagnostics $\mathrm{GmbH}$, Mannheim, Germany. Serum vitamin D was measured by enzyme-linked immunosorbent assay (ELISA). Serum lipids (triglyceride, cholesterol, low-density lipoproteincholesterol [LDL-C], high-density lipoprotein-cholesterol [HDL-C]) and serum albumin were measured using standard kits and using standard automated techniques.

Serum FGF23 (C-term) was measured using immunoenzymometric assay (IEMA) by ELISA kit (Immutopics, San Clemente, CA, USA).

\section{Statistical analysis}

Results were expressed as mean $( \pm \mathrm{SD})$ and considered statistically significant when $P<0.05$. For correlations, Pearson product-moment correlation coefficient was used. For the assessment of difference between male and female population, the student $t$-test was applied. Data were analyzed with Stata software (Stata Corp. 2011, Stata Statistical Software: Release 12, College Station, TX, Stata Corp LP).

\section{RESULTS}

A total of 61 patients (19 females, 42 males) were enrolled in this study. Mean $( \pm$ SD) age of the participants was 59.6 \pm 13 years (range: $25-90$ years). The median duration of dialysis was 23 months (range: 6-120 months). The major causes of CKD in the studied subjects were diabetes mellitus $(n=22,36 \%)$ and HTN $(n=14,23 \%)$. Table 1 shows the basic demographic characteristics of the patients.

The median of serum concentration of FGF23 was 1,977 $\mathrm{pg} / \mathrm{dL}$ (range: $155-8,870 \mathrm{pg} / \mathrm{dL}$ ). The median of FGF23 was $2074 \mathrm{pg} / \mathrm{dL}$ in males and $1,929 \mathrm{pg} / \mathrm{dL}$ in females; as a result, there was no significant difference between them $(P=0.37)$. Table 2 shows the results of relevant laboratory data and echocardiographic findings of the patients. The mean $( \pm$ SD) of LVMI measured in the patients was $152 \pm$ $52 \mathrm{~g} / \mathrm{m}^{2}$. When this index was measured separately in both genders, no significant difference $(P=0.281)$ was noticed between them; however, male subject has slightly higher LV mass $\left(157 \pm 57 \mathrm{~g} / \mathrm{m}^{2}\right)$ compared to female subjects (141 \pm $\left.38 \mathrm{~g} / \mathrm{m}^{2}\right)$. LVH was seen in $73.8 \%$ of the patients $(n=45)$ and of them $66.7 \%$ were male. In LVH group, the median of FGF23 was 2,088 pg/dL compared to $1,929 \mathrm{pg} / \mathrm{dL}$ in patients without $\operatorname{LVH}(P=0.71)$. To evaluate the impact of FGF23 on ventricular hypertrophy, the correlation between serum levels of FGF23 and echocardiographic index of ventricular function, demographic characteristics, and laboratory data of the study group was assessed (Table 3). We found a significant positive correlation between FGF23 and LVDs $(r=0.287, P=0.02)$, while no correlation was found between FGF23and LVMI $(r=$ $-0.085, P=0.52)$. Among the biochemical parameters, there was only a significant association between FGF23 and serum phosphorus $(r=0.584, P<0.001)$. The correlation

\begin{tabular}{lll}
\hline \multicolumn{2}{l}{ Table 1: Demographic characteristics of the study group } \\
\hline Variables & Mean (SD) & Median (Min.-Max.) \\
\hline Age (years) & $59.6(13.1)$ & $61(25-90)$ \\
BMI $\left(\mathrm{kg} / \mathrm{m}^{2}\right)$ & $25.1(4.9)$ & $24.8(15.2-40.2)$ \\
BSA $\left(\mathrm{m}^{2}\right)$ & $1.78(0.21)$ & $1.79(1.39-2.58)$ \\
Systolic blood pressure $(\mathrm{mmHg})$ & $128.4(21.2)$ & $125(91-188)$ \\
Diastolic blood pressure $(\mathrm{mmHg})$ & $76.3(11.5)$ & $76(46-103)$ \\
Dialysis duration (months) & $31.7(25.7)$ & $23(6-120)$ \\
Volume change (L) & $1.87(0.66)$ & $1.80(0.80-5.20)$ \\
\hline
\end{tabular}


Nassiri et al.: FGF23 in patients on hemodialysis

\begin{tabular}{|c|c|c|}
\hline Parameter & Mean (SD) & Median (Min.-Max.) \\
\hline$\overline{\mathrm{FGF} 23(\mathrm{pg} / \mathrm{dL})}$ & $2486(2391)$ & $1977(155-11,010)$ \\
\hline Hemoglobin (g/L) & $109(15)$ & $108(71-148)$ \\
\hline Albumin (g/L) & $38(4)$ & $39(27-50)$ \\
\hline iPTH (ng/L) & $241(213)$ & $182(11-919)$ \\
\hline Vitamin D (nmol/L) & $46.5(8)$ & $47(30-75)$ \\
\hline Calcium (mmol/L) & $2.0(0.17)$ & $1.98(1.52-2.52)$ \\
\hline Phosphorus (mmol/L) & $1.25(0.39)$ & $1.19(0.49-2.66)$ \\
\hline Triglyceride (mmol/L) & $1.59(0.97)$ & $1.37(0.40-4.39)$ \\
\hline Cholesterol (mmol/L) & $3.82(0.94)$ & $3.80(2.21-6.55)$ \\
\hline LDL-C (mmol/L) & $2.26(0.73)$ & $2.27(0.80-4.89)$ \\
\hline $\mathrm{HDL}-\mathrm{C}(\mathrm{mmol} / \mathrm{L})$ & $0.78(0.26)$ & $0.73(0.36-1.72)$ \\
\hline LV-Mass (g) & $271(91)$ & $276(102-545)$ \\
\hline LV-Mass index $\left(\mathrm{g} / \mathrm{m}^{2}\right)$ & $152(52)$ & $151(27-319)$ \\
\hline LVDd (mm) & $48(7)$ & $48(31-69)$ \\
\hline LVDs (mm) & $31(8)$ & $31(11-52)$ \\
\hline $\operatorname{LVEDV}(\mathrm{mL})$ & $135(49)$ & $138(13-259)$ \\
\hline $\operatorname{LVESV}(\mathrm{mL})$ & $51(26)$ & $48(12-142)$ \\
\hline LVEF (\%) & $54(11)$ & $44(30-74)$ \\
\hline FS (\%) & $24.9(7.3)$ & $35.8(18.5-59.3)$ \\
\hline $\mathrm{PWT}(\mathrm{mm})$ & $11.9(2.2)$ & $11.8(7.0-18.4)$ \\
\hline E/A ratio & $1.0(0.6)$ & $0.9(0.4-4.1)$ \\
\hline
\end{tabular}

FGF23, fibroblast growth factor 23; iPTH, intact parathyroid hormone; LDL-C, low-density lipoprotein-cholesterol; HDL-C, high-density lipoprotein-cholesterol; LV, left ventricle; LVDd, left ventricle diameter in end diastole; LVDs, left ventricle diameter in end systole; LVEDV, left ventricle volume in end diastole; LVESV, left ventricle volume in end systole; LVEF, Left ventricle ejection fraction; FS, fractional shortening; PWT, posterior left ventricular wall thickness; E/A ratio, early to late ventricular filling velocity.

between FGF23 and serum iPTH was considerable, although it did not reach a significant level $(r=0.243$, $P=0.063)$. Evaluation of association of LV mass with echocardiographic findings of the patients only showed significant association with LVDd and LVDs $(P=0.007)$. Except for iPTH that was significantly higher in LVH group (197 $\mathrm{ng} / \mathrm{L})$ than that in the patients without LVH $(132 \mathrm{ng} / \mathrm{L})(P=0.06)$, other demographic or biochemical data were similar in both groups (Table 4).

\section{DISCUSSION}

The FGF family consists of 23 members (FGF1 to FGF23); FGF23, as a novel member of this family, is a bone-derived protein, secreted mainly by osteoblasts

\begin{tabular}{lll}
\multicolumn{2}{l}{ Table 3: Correlation between FGF23 and the following } \\
characteristics & $\boldsymbol{r}$ & $\boldsymbol{P}$ value \\
\hline Characteristics & -0.092 & 0.488 \\
\hline Age (years) & 0.213 & 0.106 \\
Dialysis duration (months) & 0.114 & 0.389 \\
Volume change (L) & -0.109 & 0.412 \\
Hemoglobin (g/L) & 0.243 & 0.063 \\
iPTH (ng/L) & 0.121 & 0.361 \\
Vitamin D (nmol/L) & 0.111 & 0.401 \\
Calcium (mmol/L) & 0.584 & $<0.001$ \\
Phosphorus (mmol/L) & -0.049 & 0.710 \\
LV-Mass (g) & -0.085 & 0.522 \\
LV-Mass index(g/m²) & 0.102 & 0.441 \\
LVDd (mm) & -0.287 & 0.027 \\
LVDs (mm) & -0.059 & 0.656 \\
LVEDV(mL) & 0.155 & 0.241 \\
LVESV(mL) & 0.011 & 0.937 \\
LVEF (\%) & -0.416 & 0.001 \\
FS (\%) & -0.170 & 0.167 \\
PWT(mm) & & \\
E/A ratio & & \\
\hline
\end{tabular}

FGF23, fibroblast growth factor 23; iPTH, intact parathyroid hormone; LV, left ventricle; LVDd, left ventricle diameter in end diastole; LVDs, left ventricle diameter in end systole; LVEDV, left ventricle volume in end diastole; LVESV, left ventricle volume in end systole; LVEF, Left ventricle ejection fraction; FS, fractional shortening; PWT, posterior left ventricular wall thickness; E/A ratio, early to late ventricular filling velocity.

and osteocytes, and has a function quite different from other members of the FGF family. ${ }^{[4,5]}$ Numerous studies revealed that FGF23 as a phosphate binding hormone is independently associated with several cardiovascular risk factors. ${ }^{[3,10]}$ Thus, FGF23 could be considered as the novel risk biomarker for CVD death in patients on hemodialysis. Therefore, as CVDs are the leading cause of death in patients with $\mathrm{CKD},{ }^{[1,3]}$ in this study, we aimed to evaluate the association between serum levels of FGF23 and echocardiographic indexes of LV dysfunction in patients on hemodialysis. Our findings suggest that once relevant confounders were considered, the relation between serum FGF23 levels and LVDs was significant, while there was no significant relationship between FGF23 and LVDd, PWT, LV mass, LVMI, and LVEF. In adults with CKD, the link between FGF23 concentration and LVH has been documented in many studies; however, studies regarding the association of FGF23 with LVH in patients who are on hemodialysis are less frequent and sometimes addressed various results. 
Nassiri et al: FGF23 in patients on hemodialysis

\begin{tabular}{|c|c|c|c|}
\hline \multirow[t]{2}{*}{ Characteristics } & \multirow{2}{*}{$\begin{array}{l}\text { LVH group } \\
n=45\end{array}$} & \multirow{2}{*}{$\begin{array}{l}\text { Non-LVH group } \\
n=16\end{array}$} & \multirow[t]{2}{*}{$P$ value } \\
\hline & & & \\
\hline Age (years) & $59.1(13.3)$ & $61.1(12.8)$ & 0.598 \\
\hline BMI & $24.8(4.4)$ & $26.0(6.2)$ & 0.408 \\
\hline Dialysis duration (months) & $23(6-120)$ & $15(6-62)$ & 0.055 \\
\hline Volume change (L) & $1.8(0.5)$ & $2.1(1.0)$ & 0.338 \\
\hline FGF23 (pg/dL) & ${ }^{\square}, 088(155-8,870)$ & $1,929(256-8,169)$ & 0.715 \\
\hline Hemoglobin (g/L) & $10.8(1.6)$ & $11.2(1.3)$ & 0.394 \\
\hline iPTH (ng/L) & $197(11-919)$ & $132(21-339)$ & 0.064 \\
\hline Vitamin D (nmol/L) & $46.7(8.5)$ & $46.5(6.5)$ & 0.952 \\
\hline Calcium (mmol/L) & $2(0.1)$ & $1.9(0.2)$ & 0.882 \\
\hline Phosphorus (mmol/L) & $1.2(0.3)$ & $1.4(0.4)$ & 0.203 \\
\hline Triglyceride (mmol/L) & ${ }^{\square} 1.4(0.4-4.5)$ & $1.3(0.5-3.8)$ & 0.954 \\
\hline Cholesterol (mmol/L) & $3.8(1)$ & $3.8(0.8)$ & 0.848 \\
\hline $\mathrm{LDL}(\mathrm{mmol} / \mathrm{L})$ & $2.2(0.7)$ & $2.3(0.7)$ & 0.474 \\
\hline $\mathrm{HDL}(\mathrm{mmol} / \mathrm{L})$ & $0.8(0.3)$ & $0.7(0.2)$ & 0.158 \\
\hline LVDd (mm) & $49(7)$ & $44(7)$ & 0.007 \\
\hline LVDs (mm) & $32(7)$ & $26(7)$ & 0.007 \\
\hline LVEDV (mL) & $140(52)$ & $120(40)$ & 0.186 \\
\hline LVESV (mL) & $53(26)$ & $46(26)$ & 0.376 \\
\hline LVEF (\%) & $53(12)$ & $56(10)$ & 0.417 \\
\hline FS(\%) & $86(28)$ & $92(28)$ & 0.098 \\
\hline PWT (mm) & $86(28)$ & $92(28)$ & 0.320 \\
\hline E/A ratio & $86(28)$ & $92(28)$ & 0.620 \\
\hline
\end{tabular}

Data are shown as mean (SD) and Das median (Min.-Max.). FGF23, fibroblast growth factor 23; LVH, left ventricular hypertrophy; BMI, body mass index; iPTH, intact parathyroid hormone; LDL-C, low-density lipoprotein-cholesterol; HDL-C, high-density lipoprotein-cholesterol; LV, left ventricle; LVDd, left ventricle diameter in end diastole; LVDs, left ventricle diameter in end systole; LVEDV, left ventricle volume in end diastole; LVESV, left ventricle volume in end systole; LVEF, Left ventricle ejection fraction; FS, fractional shortening; PWT, posterior left ventricular wall thickness; E/A ratio, early to late ventricular filling velocity.

Hsu et al. examined 124 patients with end-stage kidney failure and found that LVH was correlated with higher levels of FGF23. They concluded from results that serum FGF23 level is independently associated with LVH; however, it is not a predictor for short-term prognosis (2-year follow-up). ${ }^{[22]}$ In a recent study, Kovács and colleagues ${ }^{[23]}$ by using three-dimensional speckle tracking echocardiography investigated the effects of FGF23 on LV mechanics in patients on hemodialysis and found that elevated FGF23 levels were associated with increased LV mass $(\varrho=0.581, P<0.001)$. Accordingly, Liu et al. ${ }^{[24]}$ examined the relationship between FGF23and LVH in 62 patients on continuous ambulatory peritoneal dialysis and 30 healthy controls ${ }^{[24]}$. They found that serum FGF23 level were significantly higher in patients on continuous ambulatory peritoneal dialysis than in healthy controls, while both were higher in patients with LVH than in individuals without LVH. Also, FGF23 was detected to be positively associated with LVMI. They concluded that FGF-23 is an independent risk factor for LVH in patients on continuous ambulatory peritoneal dialysis. ${ }^{[4]}$ In contrast, Knap et al. examined the possible association between FGF23 levels with LVMI in 50 patients on maintenance hemodialysis and found no correlations between log FGF23 levels and LVMI, a result that is in accordance with our findings. ${ }^{[25]}$ 
It is shown that the extent of LV dysfunction produced by heart disease is a major determinant of clinical prognosis and survival. ${ }^{[27]}$ The assessment of cardiac chamber dimensions, ventricular function, and myocardial mass is among echocardiographic indexes that have an important prognostic value. ${ }^{[19,27,28]}$ However, it should be noted that LV diameter is a relatively crude assessment of a three-dimensional structure that cannot consider for more complex variations in ventricular shape or size, ${ }^{[29-31]}$ an issue that might be the reason for not seeing an association between serum levels of FGF23 and LVM index in our study. Moreover, better phosphate level, blood pressure, and anemia control in our patients might also be considered, at least partially, as the reason for not seeing this difference. ${ }^{[32]}$

It is correct that ejection phase indexes, particularly ejection fraction, are the most used clinical parameters of LV function, but this index is affected by changes in loading conditions independent of changes in muscle function. On the other hand, end systolic volume is independent of preload, and therefore, it is recommended that end systolic volume index might be a better marker of contractile function than ejection fraction in patients in whom the preload is altered. ${ }^{[30,31]}$ This might explain why we did not see an association between FGF23 and ejection fraction while the relation was present between the growth factor and LVDs.

\section{CONCLUSION}

In summary, in our study, the association of FGF23 with LV mass, LVMI, and LVEF was not significant. We found a positive significant association of FGF23 with LVDs. However, the association of FGF23 with LV mass, LVMI, and LVEF was not significant. Additional longitudinal investigations that evaluate the predictive power of FGF23 and analyze whether FGF23 has additional clinical applications are necessary. Also, it is needed to evaluate the role of treatment strategies and reducing serum levels of FGF23 and to analyze if it has to be associated with decreased rate of cardiovascular events. ${ }^{[33]}$

\section{Conflict of Interest}

The authors declare there are no conflicts of interest.

\section{REFERENCES}

1. Go AS, Chertow GM, Fan D, McCulloch CE, Hsu C. Chronic kidney disease and the risks of death, cardiovascular events, and hospitalization. N Engl J Med 2004; 351: 1296-305.

2. Sinha MD, Turner C, Booth CJ, Waller S, Rasmussen P, Goldsmith DJ, et al. Relationship of FGF23 to indexed left ventricular mass in children with non-dialysis stages of chronic kidney disease, Pediatr Nephrol
2015; 30: 1843-52.

3. Larsson TE. FGF23 beyond Mineral Metabolism: A bridge to cardiovascular disease. Clin J Am Soc Nephrol 2011;6: 2735-7.

4. Eswarakumar VP, Lax I, Schlessinger J. Cellular signaling by fibroblast growth factor receptors. Cytokine Growth Factor Rev 2005; 16: 139-49.

5. Chong WH, Molinolo AA, Chen CC, Collins MT. Tumor-induced osteomalacia. Endocr Relat Cancer 2011;18: R53-77.

6. Ornitz DM. FGFs, heparan sulfate and FGFRs: complex interactions essential for development. BioEssays 2000; 22:108-12.

7. Goetz R, Beenken A, Ibrahimi OA, Kalinina J, Olsen SK, Eliseenkova AV, et al. Molecular insights into the klotho-dependent, endocrine mode of action of fibroblast growth factor 19 subfamily members. Mol Cell Biol 2007; 27: 3417-28.

8. Urakawa I, Yamazaki Y, Shimada T, Iijima K, Hasegawa H, Okawa K, et al. Klotho converts canonical FGF receptor into a specific receptor for FGF23. Nature 2006; 444: 770-4.

9. Kurosu H, Ogawa Y, Miyoshi M, Yamamoto M, Nandi A, Rosenblatt $\mathrm{KP}$, et al. Regulation of fibroblast growth factor-23 signaling by klotho. J Biol Chem. 2006; 281: 6120-3.

10. Gutiérrez O, Mannstadt M, Isakova T, Rauh-Hain JA, Tamez H, Shah A, et al. Fibroblast Growth Factor 23 and Mortality among Patients Undergoing Hemodialysis. N Engl J Med 2008; 359: 584-92.

11. Gutierrez O, Isakova T, Rhee E, Shah A, Holmes J, Collerone G, et al. Fibroblast growth factor-23 mitigates hyperphosphatemia but accentuates calcitriol deficiency in chronic kidney disease. J Am Soc Nephrol 2005; 16: 2205-15.

12. Isakova $\mathrm{T}$, Xie H, Yang W, Xie D, Anderson AH, Scialla J, et al; Chronic Renal Insufficiency Cohort (CRIC) Study Group (2011). Fibroblast growth factor 23 and risks of mortality and end-stage renal disease in patients with chronic kidney disease. JAMA 2011; 305: 2432-9.

13. Faul C, Amaral AP, Oskouei B, Hu MC, Sloan A, Isakova T, et al. FGF23 induces left ventricular hypertrophy. J Clin Invest 2011; 121: 4393-408.

14. Kovesdy CP, Quarles LD. Fibroblast growth factor-23: what we know, what we don't know, and what we need to know. Nephrol Dial Transplant 2013; 28: 2228-36.

15. Kestenbaum B, Sachs MC, Hoofnagle AN, Siscovick DS, Ix JH, RobinsonCohen $\mathrm{C}$, et al. Fibroblast growth factor-23 and cardiovascular disease in the general population: the multi-ethnic study of atherosclerosis. Circ Heart Fail 2014; 7: 409-17.

16. Shibata K, Fujita S, Morita H, Okamoto Y, Sohmiya K, Hoshiga M. Association between circulating fibroblast growth factor 23, a-Klotho, and the left ventricular ejection fraction and left ventricular mass in cardiology in patients. PLoS One 2013; 8:e73184.

17. du Bois D, du Bois EF. A formula to estimate the approximate surface area if height and weight are known. Arch Intern Med 1916; 17: 863-71.

18. Stolzmann P, Scheffel H, Trindade PT, Plass AR, Husmann L, Leschka $\mathrm{S}$, et al. Left ventricular and left atrial dimensions and volumes: comparison between dual-source CT and echocardiography. Invest Radiol 2008; 43: 284-9.

19. Gibson PH, Becher H, Choy JB. Classification of left ventricular size: diameter or volume with contrast echocardiography? Open Heart 2014; 1: e000147.

20. Isakova T, Xie H, Yang W, Xie D, Anderson AH, Scialla J, et al; Chronic Renal Insufficiency Cohort (CRIC) Study Group. Fibroblast growth factor 23 and risks of mortality and end-stage renal disease in patients with chronic kidney disease. JAMA 2011; 305:2432-9.

21. Gutiérrez OM, Januzzi JL, Isakova T, Laliberte K, Smith K, Collerone $\mathrm{G}$, et al. Fibroblast growth factor 23 and left ventricular hypertrophy in chronic kidney disease. Circulation 2009; 119: 2545-52.

22. Hsu HJ, Wu MS. Fibroblast growth factor 23: a possible cause of left ventricular hypertrophy in hemodialysis patients. Am J Med Sci 2009; 337:116-22.

23. Kovács A, Tapolyai M, Celeng C, Gara E, Faludi M, Berta K, et al. Impact of hemodialysis, left ventricular mass and FGF-23 on myocardial me- 
chanics in end-stage renal disease: a three-dimensional speckle tracking study. Int J Cardiovasc Imaging 2014; 30: 1331-7.

24. Liu WH, Zhou QL, Ao X, Yu HL, Peng WS, He N. Fibroblast growth factor-23 and interleukin-6 are risk factors for left ventricular hypertrophy in peritoneal dialysis patients. J Cardiovasc Med (Hagerstown) 2012; 13: 565-9.

25. Knap B, Večerić-Haler Ž, Benedik M, Buturović-Ponikvar J, Ponikvar $\mathrm{R}$, Bren AF. Fibroblast growth factor 23 and left ventricular mass index in maintenance hemodialysis patients: standard versus long nocturnal hemodialysis. Ther Apher Dial 2013; 17: 407-11.

26. Carabello BA, Spann JF. The uses and limitations of end-systolic indexes of left ventricular function. Circulation 1984; 69:1058-64.

27. Gibson PH, Becher H, Choy JB. Classification of left ventricular size: diameter or volume with contrast echocardiography? Open Heart 2014; 1: e000147.

28. Greenhill C. Risk factors: Levels of FGF23 predict outcomes in advanced CKD. Nat Rev Nephrol 2011; 7: 606.

29. Ix JH, Katz R, Kestenbaum BR, de Boer IH, Chonchol M, Mukamal KJ, et al. Fibroblast growth factor-23 and death, heart failure, and cardiovascular events in community-living individuals: CHS (Cardiovascular Health Study). J Am Coll Cardiol 2012; 60: 200-7.
30. Kendrick J, Cheung AK, Kaufman JS, Greene T, Roberts WL, Smits G, et al; HOST Investigators. FGF-23 associates with death, cardiovascular events, and initiation of chronic dialysis. J Am Soc Nephrol 2011; 22:1913-22.

31. Armstrong AC, Gidding S, Gjesdal O, WU Colin, Bluemke DA, Lima JA. LV mass assessed by echocardiography and CMR, cardiovascular outcomes, and medical practice. J Am Coll Cardiol Img 2012; 5: 837-48.

32. Toto RD. From phosphaturia to cardiovascular protection. Is FGF-23 the heart of the matter? Circulation 2015; 132:7-9.

33. Moe SM, Chertow GM, Parfrey PS, Kubo Y, Block GA, Correa-Rotter $\mathrm{R}$, et al; Evaluation of Cinacalcet $\mathrm{HCl}$ Therapy to Lower Cardiovascular Events (EVOLVE) Trial Investigators. Cinacalcet, Fibroblast Growth Factor-23, and Cardiovascular Disease in Hemodialysis: The Evaluation of Cinacalcet $\mathrm{HCl}$ Therapy to Lower Cardiovascular Events (EVOLVE) Trial. Circulation 2015; 132: 27-39.

How to cite this article: Nassiri AA, Hakemi MS, Safar-Pour R, Ahmadi A, Tohidi M, Sharif Kashani B, et al. Association of serum intact fibroblast growth factor 23 with left ventricular mass and different echocardiographic findings in patients on hemodialysis. J Transl Intern Med 2016; 4: 135-41. 